\title{
SERIES INTRO \\ Regulation of interactions between cells and extracellular matrix: a command performance on several stages
}

\author{
E. Helene Sage \\ Department of Vascular Biology, The Hope Heart Institute, 1124 Columbia Street, Suite 723, Seattle, Washington 98104, USA. \\ Phone: (206) 903-2026; Fax: (206) 903-2044; E-mail: hsage@hopeheart.org.
}

Cell behaviors that feature prominently in development and disease - shape, adhesion, migration, differentiation - have become some of the major watchwords in cell biology today. These activities are mediated by interactions of cells with an extracellular environment, consisting in most cases of a heterogeneous, macromolecular matrix that is specific, not only to a given tissue, but to cells contributing to the compartmentalization of organs and tissues. That cellmatrix communication, defined broadly as receptorligand interactions resulting in signal transduction for the initiation/modification of cell behavior, is now a dominant theme in basic biomedical research attests to the insight of embryologists working nearly one hundred years ago, as well as investigators within the last quarter-century, who showed that cell shape was linked to cell fate and cell performance (1). In a recent minireview series on integrins, predominant but not exclusive mediators of information between cells and the extracellular milieu, John McDonald recalled the concept of "dynamic reciprocity," coined originally to describe the interdependent relationship of cells to matrix, which changes with cellular differentiation or tissue remodeling (2). This Perspective series on cellmatrix interactions brings together, in a novel juxtaposition, mediators of cell shape in vivo and in vitro. The six articles, each focusing largely on one protein or proteoglycan (or family) in the context of its general effects on cell behavior as a function of extracellular matrix integrity, range in content from cell adhesion and counteradhesion, to connective tissue structure and function. However, readers will note a leitmotif throughout the articles that relates to the interpretation of extracellular signals at the cell surface.

Several years ago, Paul Bornstein turned the phrase "matricellular proteins," defined as secreted macromolecules that interact with cell-surface receptors, extracellular matrix (ECM), growth factors, and/or proteases but do not in themselves subserve strictly or exclusively structural roles (3). Originally limited to SPARC (secreted protein acidic and rich in cysteine), thrombospondin 1 (TSP1), and tenascin C, this group now includes other proteins and proteoglycans, some of which are featured in this Perspective series (TSP2, tenascin X, syndecans, osteopontin). Matricellular pro- teins (or the families to which they belong) are structurally unrelated glycoproteins that, under certain conditions, disrupt cell-matrix interactions (counteror de-adhesion) and are associated with remodeling, morphogenesis, and vascular growth $(3,4)$. Thus, the grouping is one based on function, with variability among its constituents resulting from cell/tissue specificity, pathology versus normal development, compensatory effects of other proteins, etc. The matricellular proteins, which often act as extracellular "adapters" or "interface molecules," have modular structures, the domains of which account for a pleiotropy of functions. In contrast to the severe or lethal phenotypes seen in mice with targeted deletions of, for example, VEGF, collagen I, or fibronectin, animals null for the matricellular proteins mentioned above are viable and appear superficially normal, due in part to compensatory or overlapping functions subserved by related genes. However, upon closer examination, both developmental and challenge phenotypes have become apparent in many of these animals (e.g., vascular, wound healing, bone, immunocompromise, hemostasis, and connective tissue) that confirm and extend the significant roles exerted by this group of macromolecules in the design, maintenance, and repair of most tissues.

\section{Adhesion and counteradhesion}

Huttenlocher et al. (5), in their commentary on cell migration as a function of adhesive interactions, used the term "adhesive asymmetry" to point out both the variable affinities of cell receptors for matrix ligands and the nonuniform distribution of these contact points across the cell surface. It has been known for some time that the "founding family" of matricellular proteins - SPARC, TSP1, and tenascin C - exhibited counteradhesive activity on cultured cells (4). Although several mechanisms have been identified to account for this activity, the compelling question of significance remains. The first three articles in this series address adhesion as a function of TSP1 (6), TSP2 (7), and the syndecans (8). Joanne Murphy-Ullrich discusses the intermediate levels of cell adhesion - a state between attachment (weak) and focal adhesion/stress fiber formation (strong) - and the roles and biological signifi- 


\section{Matricellular proteins}

E. Helene Sage, Series Editor cance of transitions among these states as mediated by SPARC, TSP1, and tenascin C (6). A somewhat different approach to this topic is taken by Paul Bornstein, who emphasizes the interaction of matrix metalloproteinase-2 (MMP-2) with TSP2 as a significant regulator of cell adhesion (7).

An important mechanism of counteradhesion appears to be linked to the availability and/or activity of certain matricellular proteins $(6,7,9,10)$, whose counteradhesive activity could be mediated at both intercellular (e.g., via cadherins or CAMs) and cell-ECM sites (e.g., integrin-matrix ligand). Our first reported function of SPARC was its ability to change cell shape, an effect highly pronounced in endothelial cells and fibroblasts, and virtually absent in transformed cells (9), whereas SPARC-null cells exhibit enhanced spreading and increased numbers of focal adhesion plaques (10). In contrast, David Denhardt et al. have proposed that osteopontin functions as a "survival molecule" through its interaction with one or more ECM receptors on the cell surface that transmit antiapoptotic signals (11). That this mechanism can be abrogated by extracellular proteases highlights yet another tier of complexity in our analyses of how matricellular proteins work, and could account for data that appear to be disparate. The idea that otherwise cryptic sequences can be released from native proteins by proteolysis, and that their activities are often different from those of the parent molecule, has become attractive, due in part to examples in angiogenesis (12). Davis et al. (13) have coined the term "matricryptins" to describe proteolytic fragments of ECM that contain exposed bioactive sites thought to regulate tissue response to injury. An example of a matricryptin is the $\mathrm{Cu}^{2+}$-binding peptide $\mathrm{KGHK}$, which stimulates wound healing and angiogenesis, modulates the expression of MMP-2 in wounds, and is released from SPARC by serine proteases and/or MMPs (9). Proteolytic fragments or soluble forms of osteopontin, and of certain of the syndecans and tenascins that exhibit bioactivity, have also been described (6-12).

Ann Woods's analysis of the syndecan family emphasizes the interesting connection between signaling pathways originating from two sources: via cell adhesion (through integrins) and via growth factor binding (8). That syndecans might mediate a convergence of these seminal cascades is not only exciting but provides a novel explanation for the apparent disparities or redundancies noted in adhesion-based signaling (14). Moreover, support for syndecans as molecules that regulate both growth factor and ECM functions can be derived from recent studies on the basement membrane component laminin 5 (15) and the matricellular protein SPARC (9). Although regulation of cell cycle by the matricellular group is not discussed as a primary topic in this Perspective series, the interaction of these proteins and proteoglycans with growth factors or their receptors, and the distal effects on cyclins and their associated kinases, are clearly related to states of cell adhesion (and might be responsible for some of the challenge phenotypes described below) (16).

\section{Tissue integrity and performance}

An interesting (and perhaps surprising) result in transgenic mice rendered null for several of the matricellular genes is the effect on collagen fiber morphology. Partial or full inactivation of an "accessory" collagen gene can alter collagen fibrillogenesis and the morphology and/or function of specific tissues, consequences that might become especially apparent during stages of development or responses to injury, when the need for high levels of collagen I, for example, is acute. Many if not all of the matricellular macromolecules bind to one or more types of collagen, although it has not been shown definitively that this property is responsible for the aberrant collagen fibrils reported in the dermis of mice lacking SPARC and TSP2 $(7,10)$. There is, however, precedent for collagen-binding proteoglycans affecting collagen fibrillogenesis, since the size, distribution, and assembly of collagen fibrils are abnormal in mice lacking decorin, fibromodulin, or lumican $(17,18)$. These animals also display either fragile skin phenotypes or alterations in tendon collagen fibrils. Perhaps the epitome of this phenotype is the recapitulation of the dermal aspects of the Ehlers-Danlos syndrome (characterized by skin laxity, joint hypermobility, failure of connective tissues, and blood vessel rupture) in mice deficient in the gene encoding tenascin $\mathrm{X}$, as described by Mao and Bristow in the final article of this Perspective series (19). This finding emphasizes the importance of balance and fine-tuning with respect to protein/proteoglycan concentrations in the extracellular space. Perhaps the previously unrecognized regulatory role of the matricellular components is manifested primarily in the capacity of responses to injury, as opposed to normal development, when the organization of copious amounts of secreted, structural proteins becomes necessary over a relatively short period of time.

Defects in collagen fibril assembly could also be predictive of abnormal blood vessel morphology or density, characteristics that have been apparent in TSP1-, TSP2-, and SPARC-null mice $(7,10)$. In addition, the enhanced deposition of white adipose tissue in SPARCnull mice might reflect the high degree of vascularity, as well as the prevalent connective tissue stroma, that regulates the differentiation of this tissue (10). Thus, a host of connective tissue defects, some of which could arise early in the life of the animal and/or in the absence of injury, might also be typical of mice lacking matricellular genes (19).

Further clues to the functions of the various members of the matricellular group have emerged from studies of experimental dermal wound healing in transgenic mice. Although it is useful to note the end result 
- for instance, more rapid closure of wounds in SPARC-null and TSP2-null than in wild-type mice (7, $10)$, or the slower closure seen in osteopontin-null and syndecan-1-null animals $(8,11)$ - the complexity of the wound healing response can often mask individual aspects of the process that are sensitive to a particular matricellular component or its absence. Thus, matrix deposition might be a target of syndecan-1 or -2, TSP2, SPARC, or tenascin $\mathrm{X}$, whereas the inflammatory response might be affected by TSP1 or osteopontin $(7-11,19)$. Alternatively, modulation of cell migration or infiltration, as a consequence of impaired adhesion, motility, and/or cell cycle could be attributed to most, if not all, of these proteins and proteoglycans. Rates of wound contracture might reflect the efficiency with which cells interact with provisional ECM, and the degree to which that adhesive state is modified by a matricellular protein $(6,7,10)$. Finally, the capacity of TSP 1 to activate TGF- $\beta 1$, and of SPARC to regulate TGF- $\beta 1$ expression, is expected to affect the response to injury, including inflammation $(6,7,10)$. Modulation of both the abundance and location of growth and angiogenesis factors, as well as their activation of cognate receptors, appears to be a function inherent in most of the matricellular proteins and proteoglycans described to date and constitutes one of the less explored aspects of this group of extracellular adapters.

\section{Perspectives}

A better understanding of the productive biochemical interactions that occur between the matricellular group and other proteins, e.g., structural components of the ECM, growth factors, and proteases, is clearly needed. For example, the association of collagens and other matrix proteins with matricellular proteins could be important for initiating subtle changes in cell adhesion through a type of "extracellular allostery." As extracellular adapters, matricellular proteins and especially transmembrane proteoglycans such as the syndecans have been proposed to alter signaling pathways that are otherwise activated through adhesive and growth factor interactions. Is there divergence with respect to the proximal effects of the matricellular components at the cell surface, and could these effects be linked to the activation of similar signaling molecules that produce specific responses in different cells (20)? Perhaps the study of the matricellular proteins/proteoglycans and their functions will unite the interests and efforts of investigators with expertise on both sides of the cell membrane.

\section{Acknowledgments}

I thank my colleagues who over the years have provided both ideas and data, and the scientists who contributed the articles for this Perspective series. The secretarial assistance of June Son is gratefully acknowledged.

1. Folkman, J., and Moscona, A. 1978. Role of cell shape in growth control. Nature. 273:345-349.

2. McDonald, J.A. 2000. Integrins minireview series: prologue. J. Biol. Chem. 275:21783.

3. Bornstein, P. 1995. Diversity of function is inherent in matricellular proteins: an appraisal of thrombospondin 1. J. Cell Biol. 130:503-506.

4. Sage, E.H., and Bornstein, P. 1991. Minireview. Extracellular proteins that modulate cell-matrix interactions: SPARC, tenascin, and thrombospondin. J. Biol. Chem. 266:14831-14834.

5. Huttenlocher, A., Sandborg, R.B., and Horwitz, A.F. 1995. Adhesion in cell migration. Curr. Opin. Cell Biol. 7:697-706.

6. Murphy-Ullrich, J.E. 2001. The de-adhesive activity of matricellular proteins: is intermediate cell adhesion an adaptive state? J. Clin. Invest. 107:785-790

7. Bornstein, P. 2001. Thrombospondins as matricellular modulators of cell function. J. Clin. Invest. In press.

8. Woods, A. 2001. Syndecans: transmembrane modulators of adhesion and matrix assembly. J. Clin. Invest. In press.

9. Brekken, R.A., and Sage, E.H. 2000. SPARC, a matricellular protein: at the crossroads of cell-matrix communication. Matrix Biol. 19:569-580.

10. Bradshaw, A.D., and Sage, E.H. 2001. SPARC, a matricellular protein that functions in cellular differentiation and tissue response to injury. J. Clin. Invest. In press.

11. Denhardt, D.T., Noda, M., O’Regan, A.W., Pavlin, D., and Berman, J.S. 2001. Osteopontin: a means to cope with environmental insults. Regulation of inflammation, tissue remodeling, and cell survival. J. Clin. Invest. In press.

12. Sage, E.H. 1997. Pieces of eight: bioactive fragments of extracellular proteins as regulators of angiogenesis. Trends Cell Biol. 7:182-186.

13. Davis, G.E., Bayless, K.J., Davis, M.J., and Meininger, G.A. 2000. Regulation of tissue injury responses by the exposure of matricryptic sites within extracellular matrix molecules. Am. J. Pathol. 156:1489-1498.

14. Fashena, S.J., and Thomas, S.M. 2000. Signalling by adhesion receptors. Nat. Cell Biol. 2:E225-E229.

15. Nguyen, B.P., Ryan, M.C., Gil, S.G., and Carter, W.G. 2000. Deposition of laminin 5 in epidermal wounds regulates integrin signaling and adhesion. Curr. Opin. Cell Biol. 12:554-562.

16. Raines, E.W. 2000. The extracellular matrix can regulate vascular cell migration, proliferation, and survival: relationships to vascular disease. Int. J. Exp. Pathol. 81:173-182.

17. Danielson, K.G., et al. 1997. Targeted disruption of decorin leads to abnormal collagen fibril morphology and skin fragility. J. Cell Biol. 136:729-743

18. Ezura, Y., Chakravarti, S., Oldberg, A., Chervoneva, I., and Birk, D.E. 2000. Differential expression of lumican and fibromodulin regulate collagen fibrillogenesis in developing mouse tendons. J. Cell Biol. 151:779-788.

19. Mao, J.R., and Bristow, J. 2001. The Ehlers-Danlos syndrome: not just for collagens anymore! J. Clin. Invest. In press.

20. Kolch, W. 2000. Meaningful relationships: the regulation of the Ras/Raf/MEK/ERK pathway by protein interactions. Biochem. J. 351:289-305. 